Itinéraires Itinéraires

Littérature, textes, cultures

2010-2 | 2010

Les blogs

\title{
Du blog aux places d'Italie : le cas de Beppe Grillo et du V-Day
}

\section{Giovanna di Rosario}

\section{(2) OpenEdition}

Journals

Édition électronique

URL : http://journals.openedition.org/itineraires/2022

DOI : $10.4000 /$ itineraires.2022

ISSN : 2427-920X

Éditeur

Pléiade

\section{Édition imprimée}

Date de publication : 1 juillet 2010

Pagination : 113-120

ISBN : 978-2-296-12012-9

ISSN : $2100-1340$

\section{Référence électronique}

Giovanna di Rosario, «Du blog aux places d'Italie : le cas de Beppe Grillo et du V-Day », Itinéraires [En ligne], 2010-2 | 2010, mis en ligne le 01 juillet 2010, consulté le 02 mai 2019. URL : http:// journals.openedition.org/itineraires/2022 ; DOI : 10.4000/itineraires.2022

\section{(ब) $(\Theta \Theta$}

Itinéraires est mis à disposition selon les termes de la licence Creative Commons Attribution - Pas d'Utilisation Commerciale - Pas de Modification 4.0 International. 


\title{
Du blog aux places d'Italie : le cas de Beppe Grillo et du V-Day
}

\begin{abstract}
If Second Life can be considered as a form of virtualization of the socio-cultural sphere, Beppe Grillo's blog can be thought of as a realization of socio-political life in Italy through the Internet. This article aims at relating the socio-political event of V-Day in Italy, proposed and sponsored by the Italian comedian Beppe Grillo through his blog. Italian politics (the " casta ») seems to be afraid of the blogger and is seeking, by law, to make it difficult to publish blogs.
\end{abstract}

Keywords : blog, Italy, virtual space, real space, political life Mots clés : blog, Italie, espace virtuel, espace réel, vie politique

Les blogs sont partout, leur hétérogénéité est évidente, on le sait. Cependant, qu'est-ce exactement qu'un blog? La discussion au sujet de la définition exacte d'un blog est toujours ouverte. Des blogs ont été décrits comme des

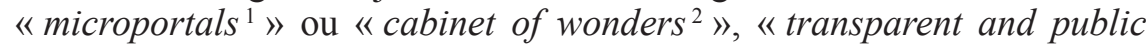
web-based soapbox and feedback system ${ }^{3} »$. La plupart des définitions contiennent l'idée qu'un blog est un site web qui est fréquemment mis à jour, où les derniers messages édités occupent le début de la page. On peut voir dans le développement de ce genre une forme de communication qui se rattache à la vision de Tim Berners-Lee, créateur du Web : «The vision I have for the web is about anything being potentially connected with anything ${ }^{4}$. » Le Web devrait être un endroit dans lequel chacun peut parler

1. Cameron Barrett, «Anatomy of a weblog », dans John Rodzvilla (éd.), We've got blog : How weblogs are changing our culture, Cambridge, Mass., Perseus Publishing, 2002, p. 25-33.

2. Julian Dibbell, «Portrait of the blogger as a young man », dans John Rodzvilla (éd.), op. cit., p. 69-77.

3. Joanne Jacobs, «Communications over exposure: the rise of blogs as a product of cybervoyeurism », ANZCA 03 Conference, Brisbane, Australia, 2003.

4. «Ma vision du Web est que tout peut être potentiellement en relation avec tout. » Tim 
sans frontières, esquivant notamment les frontières habituelles des médias traditionnels. Ito ${ }^{5}$ considère que les outils de publication comme les blogs pourraient contribuer à une nouvelle forme de démocratie dans laquelle le consensus n'est plus construit par les médias, mais par un processus de conversations entre les personnes pour atteindre le consensus.

Même si d'autres typologies existent toujours, la grande majorité des blogs sont plus narratifs et se rapprochent du genre du journal intime ${ }^{6}$; cependant, au cours des dernières années, les blogs sont devenus de plus en plus un lieu de discussion de la vie publique d'un pays (le président des États-Unis Barak Obama a bien utilisé les possibilités du blog dans sa campagne électorale).

En somme, les blogs peuvent être employés pour la réflexion sur trois domaines : le domaine subjectif (journal intime), le domaine objectif pour partager la connaissance et le domaine intersubjectif pour la critique de la société. C'est le cas du blog de Beppe Grillo ${ }^{7}$ qui est l'objet de cette étude.

Le blog de Grillo est un cas assez singulier en Italie, mais aussi plus globalement, puisqu'il montre des spécificités difficiles à retrouver dans d'autres blogs. On analysera surtout trois aspects généraux en tenant compte de la singularité proposée par le blogueur italien :

- la pratique de lecture et l'activité du lecteur;

- le rapport à la temporalité et à la spatialité;

- l'édition : le blog évite la sanction éditoriale; il semble être privé de système de légitimation. En Italie, le gouvernement Prodi, et maintenant le gouvernement Berlusconi, cherchent à censurer la plupart des blogs, ce qui leur confère au contraire une légitimité.

\section{Le blog de Beppe Grillo}

Beppe Grillo est né le 21 juillet 1948 dans la province de Gênes. C'est un comique italien et un blogueur. Tour à tour acteur de théâtre et de télévision, surtout publique, il est écarté de cette dernière en 1987 lorsqu'il attaque par de lourdes allusions le Parti socialiste italien et son chef, Bettino Craxi, à l'époque président du Conseil des ministres. Quelques années plus tard, ses allusions seront reconnues et avérées par la magistrature, notamment dans le cas judiciaire, très connu, des «Mani pulite ${ }^{8} »$.

Berners-Lee et Mark Fischetti, Weaving the Web : the original design and ultimate destiny of the World Wide Web, New York, Harper Collins, 1999, p. 1-2.

5. Joichi Ito, « Weblogs and emergent democracy », 10 août 2004, http://joi.ito.com/static/ emergentdemocracy.html, consulté le 12 avril 2010.

6. Susan Herring, Lois Scheidt, Sabrina Bonus et Elijah Wright, Bridging the Gap : A Genre Analysis of Weblogs, 2004, http://web.archive.org/web/20041204182536.

7. http://www.beppegrillo.it/.

8. Mani pulite («Mains propres») est le nom d'une opération judiciaire lancée en 1992 contre la corruption du monde politique italien et qui aboutit à la disparition du parti politique le plus important d'Italie, la Démocratie chrétienne (DC) et d'autres partis très importants 
Il y a quelques années, j'ai commencé un spectacle en y détruisant un ordinateur, mais désormais $\mathrm{j}$ 'ai compris un certain nombre de choses sur Internet, qui est notre seule défense. (B. Grillo)

Son blog d'opinion est le plus visité en Italie. Il se retrouve même dans les vingt premiers au niveau mondial selon Technorati ${ }^{9}$, le moteur de recherche américain spécialisé dans le domaine des blogs; c'est le seul blog italien de la liste, et il a été en outre classé parmi les dix blogs les plus influents au monde par The Guardian (mars 2008). Selon la recherche de Technorati, la blogosphère italienne est caractérisée par des blogs à caractère intime, des sortes de journaux intimes où les blogueurs privilégient des réflexions de nature personnelle.

Le blog de Grillo représente donc une singularité dans le panorama italien. De plus, il faut considérer, pour fondamentalement comprendre l'importance et le pouvoir du blogueur italien, la réalité informatique italienne. Selon les dernières statistiques publiées par Eurostat, le pourcentage de la population italienne qui a accès à Internet est de $43 \%$, contre, par exemple, $79 \%$ en Allemagne et $77 \%$ au Royaume-Uni ${ }^{10}$.

Le premier élément significatif dans le blog de Grillo est la présence soit du nom, soit du visage de l'auteur : en haut, à gauche on trouve écrit : « il blog di Beppe Grillo »; à droite figure le visage du comique. L'auteur signe donc de son nom mais affiche surtout sa face ${ }^{11}$ comme pour signifier qu'il prend ainsi le risque de la perdre pour ce qu'il va dire dans son blog.

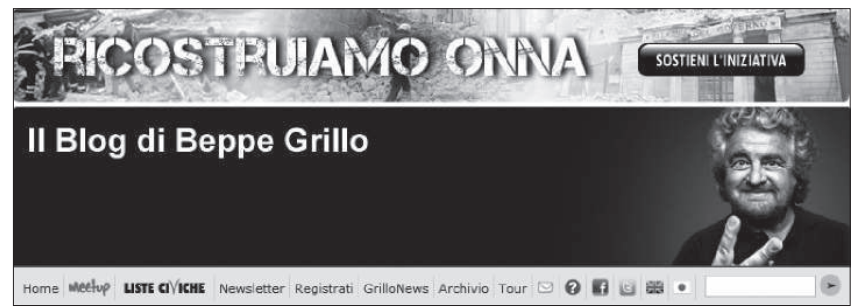

Fig. 1 - Le blog de Grillo

La mise en page du blog est assez pleine, il n'y a pas d'espace vide, ce qui signale déjà au lecteur un blog très actif.

Ce blog existe depuis cinq ans et depuis 2005 il existe une version en anglais et maintenant une version en japonais. Une équipe de cinq personnes environ, plus l'auteur, tiennent à jour le blog. Il ne s'agit pas d'un blog d'amateur. Sa fonction est de se proposer comme une alternative à l'information

pour la scène politique italienne comme le Parti socialiste (PSI) dont le secrétaire était Premier ministre en 1992.

9. Technorati, 2008 : http://technorati.com/, consulté le 12 avril 2010.

10. Eurostat, 8 décembre 2009, http://epp.eurostat.ec.europa.eu/portal/page/portal/product_details/publication?p_product_code=KS-QA-09-046, consulté le 12 avril 2010.

11. Erving Goffman a montré l'importance sociale de cette notion de «face » (Les Rites d'interaction, Paris, Éditions de Minuit, 1974, p. 9 sq.). 
« classique » qui représente pour Grillo l'information du pouvoir (et en Italie, malheureusement, c'est là un vrai souci).

Ce blog est normalement mis à jour chaque soir. Les commentaires concernent des faits tirés de la réalité sociopolitique actuelle (italienne mais pas seulement). Les articles sont quasiment toujours écrits par Grillo, mais il y a aussi des articles d'autres journalistes ou d'autres personnalités de la vie publique italienne, et même des gens ordinaires, invités par Grillo à poster des commentaires. Favorisant le travail collectif et l'échange autour d'un sujet spécifique, ce blog se positionne aussi comme une nouvelle ressource documentaire, véhiculant des informations qui s'avèrent précieuses pour les internautes qui y trouvent beaucoup d'opinions (sociales, politiques, scientifiques).

Les activités sponsorisées par Grillo sont multiples, de la class-action contre Telecom Italia, l'usine de téléphonie qui a volé plusieurs petits actionnaires italiens, au cas Aldovrandi, jeune garçon tué par la police dans des circonstances étranges : grâce à la visibilité donnée par le blog, la police a finalement commencé à faire une enquête sérieuse sur ce cas. Mais les activités les plus intéressantes sont les deux «V-Day ${ }^{12} »$, que Grillo a organisés en septembre 2007 et en avril 2008, et qui seront présentés un peu plus loin.

Un blog est avant tout un outil de publication permettant à n'importe qui de mettre des textes en ligne aussi facilement qu'il le ferait avec un logiciel de traitement de texte. Les blogs se distinguent des forums en ce qu'ils sont initialement « des espaces de publication et non de discussions ${ }^{13}$ ». Dans le blog de Grillo, on publie et on discute. La colonne vertébrale de son blog

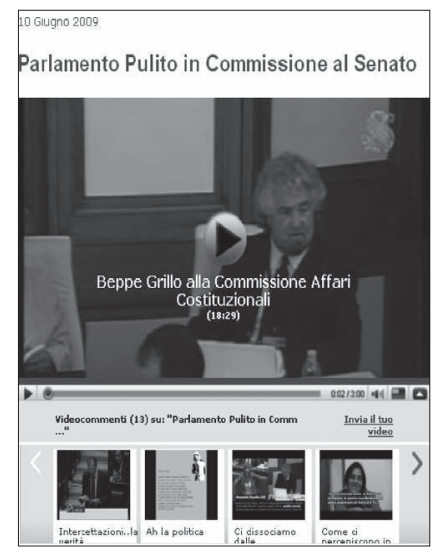

Fig. 2 - Le blog de Grillo

12. «V-Day » signifie « vaffanculo day » (« va te faire foutre »).

13. Olivier Ertzscheid, «weblogs : un nouveau paradigme pour les systèmes d'information et la diffusion de connaissances? Applications et cas d'usage en contexte de veille et d'intelligence économique », dans Organisation des connaissances dans les systèmes d'informations orientés utilisation: contexte de veille et d'intelligence économique, colloque Isko-France, 28-29 avril 2005. 
est constituée de commentaires et non de billets, même si ceux-ci sont un instrument significatif pour le blog.

\section{Lecture, spatialité, temporalité}

Dans certains types d'écriture électronique, le lecteur intervient sur la création (interactivité) : il ne se limite pas à évaluer (pratique de la réception); il participe, il propose, il est invité à interagir avec les autres lecteurs. Dans ce blog, on peut ainsi choisir de participer à la discussion, en utilisant un pseudonyme ou son propre nom. Poster est simple. Normalement, la plupart des billets sont signés par des pseudonymes. Cela signifie que la plupart des utilisateurs veulent rester anonymes : la prudence les incite à ne pas prendre totalement en charge la responsabilité de leurs mots et de leurs idées.

Le blog de Grillo n'exerce pas de censure préventive, ce sont donc les internautes qui, le cas échéant, dénoncent les billets à censurer. De plus, les internautes ont la possibilité de voter pour les billets, le billet jugé le meilleur suivra alors le commentaire de Grillo.

Mais la prise en charge de la responsabilité des idées et des opinions s'effectue plutôt hors blog. Le blog est en effet relié à des Meetups. Présents partout, même à l'étranger, ce sont des sortes d'associations (normalement formées de jeunes) qui ont une action sur le territoire : ils informent les citoyen-ne-s sur des sujets comme le recyclage des ordures, les incinérateurs ou, plus récemment, la question du nucléaire, etc. Tous les Meetups ont des blogs (sous-blogs du blog mère) où l'information circule. Mais la nouveauté consiste surtout à donner à ces blogs un prolongement hors du Net : les Meetups organisent des rendez-vous où ils invitent les citoyenne-s à participer, ils informent la population d'une manière simple, face à face. Par exemple, suite au problème des ordures à Naples (problème qui, en réalité, n'est toujours pas résolu...), les jeunes du Meetup de Naples ont organisé le 23 février 2008 une manifestation sur la Piazza Dante pour enseigner comment faire du recyclage : trente mille personnes y ont assisté. Aucun journal national (ni le Corriere della Sera, ni La Repubblica) n'a rapporté la nouvelle.

Les Meetups constituent la première forme d'expérimentation de cette sortie de la place virtuelle vers la place réelle, à laquelle tend le blog de Beppe Grillo. Et ce sont encore les Meetups qui aident Grillo dans ses deux « V-Day ».

Il y a eu deux « V-Day ». Le premier a eu lieu le 8 septembre 2007 à Bologne : la date ne relève pas du hasard, bien au contraire, car le 8 septembre 1943 est le jour de l'armistice, où le roi et l'état-major laissent l'Italie sans commandement. Un « parlement propre » y a été demandé - il y avait alors vingt-cinq parlementaires avec des condamnations définitives - et une loi populaire a été proposée. L'autre « V-Day »s'est déroulé le 25 avril 2008 à Turin : le 25 avril correspond à la fête de la libération du nazi-fascisme. Cette fois, on y a proposé de signer un référendum contre le financement 
de la presse italienne par de l'argent public - on finance même les journaux des Berlusconi! En un jour, 1500000 signatures ont été recueillies dans toute l'Italie et 150000 personnes étaient sur place à Turin pour manifester. Il faut souligner que ni la télévision ni la presse n'avait rendu compte de l'événement. Ces événements se sont passés dans le silence absolu des autres médias. Grâce à Internet et au pouvoir du blog de Grillo, les manifestations ont cependant eu un succès imprévu, inattendu pour Grillo lui-même, et causant la panique dans les médias « classiques », qui ont férocement attaqué le comique, angoissés à l'idée de perdre leur contrôle sur l'information.

Le blog de Grillo peut être défini comme réticulaire, puisqu'il a notamment des liaisons vers ses « sous-blogs » (ceux des Meetups), vers d'autres blogs et vers la place réelle, physiquement située dans la cité. Dans ce blog, il existe une sorte de démocratie partagée de l'espace d'écriture : Grillo apporte son nom, sa photo, ses idées, mais n'importe qui peut poster et on a vu qu'il n'y a pas de censure préventive. De plus, avec les Meetups, cette démocratie se partage dans la société civile.

Le blog de Grillo est un blog particulier, on pourrait le définir comme un blog performatif en ce qu'il met en acte ses idées, ses commentaires : quand dire c'est faire. Il écrit, il partage ses idées, il les met en acte. Ce blog renvoie à une spatialité et à une temporalité strictement liées l'une à l'autre : on se donne des rendez-vous, on se donne un projet, on le réalise physiquement dans des lieux publics. Les rapports que ce blog (et les Meetups) propose avec la spatialité sont donc nouveaux. On utilise l'espace virtuel pour informer, pour proposer, puis on sort de l'écran et on revient dans un espace physique, enfin on retourne au blog pour informer sur ce qui a été fait. Grâce aussi à la mise à jour quotidienne du blog, aux événements organisés par Grillo (colloques, séminaires, V-Day, «passaparola », etc.), aux Meetups, ce blog est un blog conjugué au présent : hic et nunc. Il s'inscrit dans la deixis je-ici-maintenant, c'est un work in progress qui, toutefois, souligne l'importance de la mémoire. On peut y retrouver tous les billets écrits par Grillo. Par ailleurs, les activités plus importantes ont des liens directs. Son medium ne permet pas l'oubli, sa mise en page non plus.

\section{L'édition}

En Italie, on connaît l'honnêteté de Beppe Grillo (même si la presse italienne a cherché, plusieurs fois, à détruire sa crédibilité). Le blog de Grillo est un espace dédié à la contre-information, sa légitimation est donnée par son auteur, par son histoire, par son nom et par son portrait. La mise en page du blog, colorée, intense, pleine, assez ironique, à la fois satirique et engagée, représente l'histoire du comique italien. Comme on l'a vu, Grillo poste chaque jour un billet. La communication dans ce cas reste monodirectionnelle : Grillo poste, les internautes discutent. 
Plusieurs chercheurs ont relié la théorie d'Habermas sur « l'espace public ${ }^{14} \gg$ à ce qu'on appelle la blogosphère, le réseau des blogueurs ${ }^{15}$. Selon Habermas, l'espace public est un espace de la vie sociale dans lequel l'opinion publique peut être formée. Ce domaine est accessible à toutes les personnes. Une partie de l'espace public est constituée par les conversations qui ont lieu toutes les fois que des gens se rencontrent et forment un public ${ }^{16}$.

Dans le blog de Grillo, d'une part la conversation est imposée par Grillo lui-même qui poste ses commentaires en suivant sa logique (non forcément liée aux événements du jour), d'autre part il n'intervient ni pour modérer ni pour répondre aux billets des internautes, qui en revanche animent les blogs. De toute façon, le blog de Grillo est en train de proposer un nouvel espace de discussion, qui abandonne l'espace virtuel du Web pour s'insérer dans le domaine physique de l'espace public. Les deux journées consacrées au « VDay » (et la troisième qu'il va proposer) le démontrent, de même que toutes les manifestations organisées par les Meetups, ou encore les listes civiques proposées et sponsorisées par le blog, qui ont obtenu des résultats intéressants aux dernières élections administratives du 6 et 7 juin 2009. La particularité de ce blog est d'être réticulaire dans le Web mais aussi dans le territoire.

Les objectifs que Grillo vise à rejoindre se résument à la création d'une nouvelle classe politique. Selon Transparency International (2009), l'Italie est le pays le plus corrompu de l'Europe des quinze après la Grèce. Dans le monde, elle occupe la $41^{\mathrm{e}}$ place après le Botswana et Macao ${ }^{17}$.

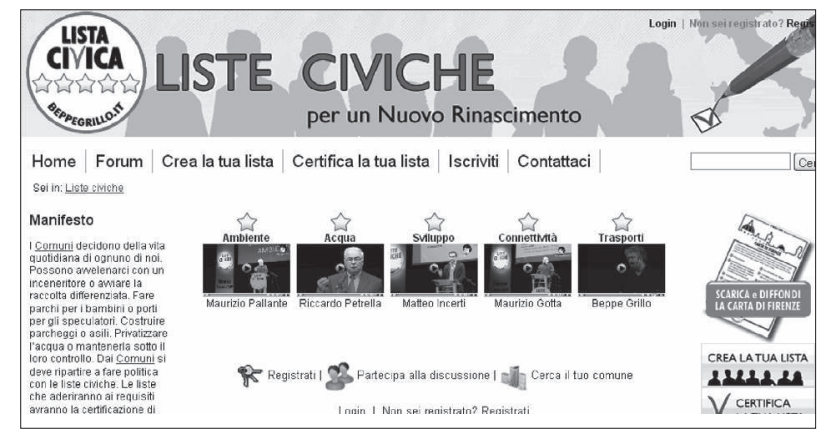

Fig. 3 - Les listes civiques

14. Jürgen Habermas, The Theory of Communicative action, vol. I, Reason and the Rationalization of Society, trad. T. McCarthy, Boston, Beacon Press, 1984.

15. Torill Mortensen et Jill Walker, «Blogging thoughts : personal publication as online research tool », dans Andrew Morrison (ed.), Researching ICTs in Context, Oslo, InterMedia Report, Université d'Oslo, 2002.

16. Gary Thompson, «Visual factors in constructing authenticity in weblogs », Toronto, Canada, 2003, http://web.archive.org/web/20040913221548/http://www.aoir.org/members/ papers42/Thompson_visauthdraft.htm, consulté le 12 avril 2010.

17. Transparency International 2009, voir Orizzontinuovi.org, http://oknotizie.virgilio.it/ go.php?us=48541240d63ca183, consulté le 15 avril 2010. 


\section{Conclusion (la censure)}

La légitimation de Grillo et de son blog comme outil pour tenter une sorte de révolution morale en Italie est si claire que l'ancien gouvernement avait proposé un projet de loi (Levi-Prodi) qui concernait la sanction éditoriale des blogs, qui permette en effet de censurer les blogs. En bref, cette loi prévoyait l'inscription des blogs dans un registre, le «Registro degli Operatori di Comunicazione (ROC) », opération assez compliquée et qui plus est contraire à la logique du blog qui voit son succès dans la facilité d'accès et d'usage. Le nouveau gouvernement Berlusconi veut proposer lui aussi une loi restrictive pour les blogs, qui sont en effet le seul moyen d'information que Berlusconi ne contrôle pas : la guerre contre Grillo et son blog a été déclarée. Le gouvernement Berlusconi vient encore d'approuver une loi qui limite l'information. Cette loi, qui empêche les interceptions, ne permettra plus de publier des nouvelles sur la base des actes judiciaires. Cela correspond évidemment à de la censure. Même Google a protesté et la question a finalement abouti sur son blog officiel. Marco Pancini, responsable pour les relations institutionnelles de Google ${ }^{18}$ en Italie, souligne que, selon cette loi, un blogueur sera considéré responsable de la même façon qu'un directeur de n'importe quel quotidien national, puisque cette loi parle généralement de « sites informatiques » (donc des blogs, mais aussi des réseaux sociaux comme Facebook, Youtube, etc.). On peut donc parler de censure, d'e-censure (censure électronique ou numérique), de fascisme et d'e-fascisme. Reste à voir la manière dont le blog de Grillo entamera le combat contre cette forme d'e-fascisme que l'Italie est en train d'expérimenter.

Giovanna di Rosario IN3-Internet Interdisciplinary Institute - HERMENEIA Research Group

18. Repubblica.it, 14 juin 2009, http://www.repubblica.it/2009/06/sezioni/politica/ddl-sicurezza-6/caselli-intercettazioni/caselli-intercettazioni.html, consulté le 12 avril 2010. 